\title{
Applications of Polypyrrole/Polyester Textiles: A Review
}

\section{Amelia Carolina Sparavigna ${ }^{1}$}

\author{
${ }^{1}$ Department of Applied Science and Technology, Politecnico di Torino, Torino, Italy
}

\begin{abstract}
The polypyrrole, in its intrinsically conducting form, can be used to prepare composites such as polypyrrole/polyester textiles by coating yarns or fabrics made of polyester. In this manner, we obtain textiles that can be involved in several applications, among which we find the use of textiles in electromagnetic shielding. However, several other uses of polypyrrole/polyester composites are possible, such as in biomedical applications. Here we propose a survey of the literature about these composites.
\end{abstract}

Keywords: Textiles, Polypyrrole, Intrinsically Conducting Polymers, Electromagnetic shielding

\section{Introduction}

When opportunely processed, the polypyrrole becomes an intrinsically conducting polymer. This material can be used to prepare polypyrrole/polyester textile composites, by coating yarns or fabrics made of polyester. In this manner we obtain textiles that can be used for several applications. Two of them are the electromagnetic interference (EMI) shielding and the radiation-absorbent camouflage [1-5]. Materials for EMI shielding are needed due to the interference of radio frequency and microwave devices with computers, transformers, cables, mobile phones, medical devices, and also for the effect of the radiation on human health. Applications of textiles is therefore required for the shielding of persons, devices or large volumes such as those of an entire room. In the case that textiles are applied as radiation-absorbent materials they can be used in stealth technology, to disguise devices or structures from radar detection. Other applications of the polypyrrole/polyester fibres and fabrics are possible, in particular in the biomedical area.

Here we propose a survey of the literature on polypyrrole/polyester textile composites. First, let us give a short discussion of both the polymers.

\section{Polyester and polypyrrole}

Polyethylene terephthalate, commonly abbreviated as PET, is a thermoplastic polymer of the polyester family. The majority of PET production is for synthetic fibers and bottles [6]. In the context of the textile applications the polymer is referred to "polyester", whereas "PET" is generally used in relation to the containers of liquids and foods and for packaging in general.
Polyester consists of polymerized units of the monomer ethylene terephthalate. According to its processing and thermal history, polyester can be an amorphous or semi-crystalline polymer. In the first case, the material is transparent; in the last case, the material can be transparent or opaque, depending on its crystal structure. From polyester threads or yarns, a variety of woven, non-woven and knitted fabrics can be obtained. Among the several properties of these polyester fabrics, let us remember that they are highly stain-resistant [7].

In the abovementioned application of textiles for electromagnetic shielding, the polyester can be coated by a conducting polymer, such as the polypyrrole (PPy). Polypyrrole is an organic polymer formed by the polymerization of pyrrole. The conductive forms of PPy are prepared by oxidation, that is, by means of a "p-doping" of the polymer. This doping creates holes (lost electrons) in the main chains of the polymer, which renders the conducting polypyrrole a "p-type" conducting material.

In this manner, the polypyrrole turns into its conductive form, becoming a conducting polymer, such as the polythiophene, polyaniline, and polyacetylene polymers [8]. Some of the first examples of conducting polypyrrole were reported in 1963 by Weiss and coworkers [9]. Let us remember also that, for the works on conducting polymers, the Nobel Prize in Chemistry was awarded in 2000 jointly to Alan J. Heeger, Alan G. MacDiarmid and Hideki Shirakawa [10-12].

This article is published under the terms of the Creative Commons Attribution License 4.0

Author(s) retain the copyright of this article. Publication rights with Alkhaer Publications.

Published at: http://www.ijsciences.com/pub/issue/2017-08/

DOI: 10.18483/ijSci.1270; Online ISSN: 2305-3925; Print ISSN: 2410-4477 
Let us survey the literature on polypyrrole/polyester fabrics starting from its applications for electromagnetic (EM) shielding.

\section{Materials for EM shielding}

Many materials had been developed for EM shielding, to protect humans and devices from radiations, in particular from electromagnetic fields. It is easy to understand why these materials are important. We have, for instance, the interference problems occurring in wireless and wired communications for the presence of large generators of radio frequency $(\mathrm{RF})$. The generated energy can affect the controls and monitoring systems of the medical facilities, for instance, and this can cause a malfunction risk to sensitive patients and laboratory equipment. Besides the evident problems of such a kind, it is the same ubiquitous presence of electronic devices which requires an investigation of their influence on the human health [13].

Shields are used to isolate a space (a room, an apparatus, a circuit etc.) from outside sources of electromagnetic radiation or prevent emissions of electromagnetic energy radiated by internal sources, so that the radiations can be reduced to an acceptable level [14]. Metallic materials are the best for any shielding purpose, but polymers are better for practical and cost effective devices. Unfortunately most polymer composites are transparent to EM radiations and suffer electrostatic discharge (ESD) [15]. To avoid EM interference and ESD, conductive polymer composites had begun to replace the use of metals, especially for the electronic housing materials. This trend had been driven mainly by the characteristics of these polymers concerning their thermal, mechanical and chemical (corrosion and oxidation resistance) properties.

\section{Metallized polyester textiles and fabrics made of hybrid yarns}

Also in the case of textiles, which require flexibility and relatively low production costs [16], the use of conductive polymers for coating fabrics or yarns can be relevant. However, let us note that for shielding EM radiations, several metallized textile fabrics exist. They are created in industrial scale processes where textile screens are covered with metals, mainly by chemical methods. Copper, nickel, silver and combinations thereof are most frequently used for the metallisation. Let us note that the shielding efficiency of these metallised fabrics derives from the energy reflection caused by the screens, and not from the absorption of EM radiations, which can be obtained by using the polypyrrole, as we will explain in the following discussion.

Another approach to EMI shielding is that of incorporating electrically conductive fillers into synthetic fabrics, injecting them during the moulding stage. Various conductive fillers are selected for different end-use depending on their shielding effectiveness (SE), at different electromagnetic frequencies. Fillers such as conductive carbon black, carbon fibres, metallized fibres, metal fibres (stainless steel, aluminium, copper), metal powders and flakes ( $\mathrm{Al}, \mathrm{Cu}, \mathrm{Ag}, \mathrm{Ni}$ ) are added to the insulating material used, or conductive yarns are incorporated into the fabric. As the fibres are closely spaced, the continuous conductive paths can be easily established, increasing the shielding properties [1719]. In the Appendices $A$ and $B$, we will propose short discussions of mechanisms behind the electromagnetic shielding and of the physical quantities used to evaluate the attenuation produced by shields.

\section{Shielding effectiveness}

Actually, several electrically conductive textiles, that are light and flexible, and in some cases able to withstand harsh environmental conditions, are available for different applications. These textiles can be divided in four main groups according to the method used for giving them conducting properties. We have metal coated fabrics, metal interwoven fabrics, filled materials, and conductive polymers, having different EMI shielding properties. Since the use of polypyrrole/polyester fabrics for EMI shielding has to be compared to that of the metallized polyester fabrics, let us shortly discuss the metallized fabrics.

To have very high degrees of shielding effectiveness, some metal coating techniques have been actually produced. The common coating methods are based on metal foils, conductive paints, sputtering coating, and vacuum depositions [20-22]. A more recent, but widely used technique, is that of the electroless plating, giving coherent metal deposition and excellent conductivity and shielding effectiveness (electroless plating is a non-electrolytic deposition by solution). In [23], a research team reported the role of surfactant concentration, acid etching, activators and so on in the multistep electroless processes for coating with this technique a polyester fabric. Other references of this technique for polyester are in Refs. 24-27. Actually, metallizing textiles is now on an industrial scale, so that several industrial metallised shielding products are available. Among them we find the Flectron, which is a Nickel/Copper coated polyester fabric [28]. The shielding effectiveness of this material ranges between $70 \mathrm{~dB}$ and $80 \mathrm{~dB}$ from $200 \mathrm{MHz}$ to $10 \mathrm{GHz}$.

A theoretical model describing the shielding effectiveness of a metallized fabric was proposed by Arthur Henn et al. [29,30]. If the wavelength of the EM radiation incident on a fabric is larger than the 
opening of the fabric, the metallized fabric will appear to that radiation as a thin metallic foil and then attenuates the signal accordingly. At higher frequency, the aperture size comes to be relevant on the overall shielding effectiveness. Thus, a metallised fabric can be modelled as a convenient thin metal foil and irregular meshes.

In [31], we find an experimental study of the electromagnetic shielding effectiveness using metal coating films and a vacuum evaporation deposition technique with the woven fabrics made of metallized polyester filaments. Using coaxial transmission equipment, SE of these films and fabrics was measured. The experimental results indicate that different coating materials have different SE, with a thicker coating film having a higher SE. In [32], electromagnetic shielding characteristics of woven fabrics made of hybrid yarns were investigated. Measurements made in the frequency range of 30 MHz-9.93 GHz give that the considered woven fabric samples have 25-65 dB SE. The paper is also showing that the direction, density and settlement type of conductive hybrid yarn in fabric structure are important parameters affecting electromagnetic shielding characteristics of woven fabrics. In [33], stainless steel was selected as the conductive filler to produce stainless steel hybrid yams to make woven fabrics (in fibres or monofilament).

\section{PPy/polyester textiles}

As previously told, intrinsically conducting polymers (ICPs) are organic polymers that conduct electricity. These compounds may have metallic conductivity or can be semiconductors, having therefore a possible use in a wide range of industrial markets [34]. Electronic markets for ICPs include, as we have told in the introduction, electromagnetic interference shielding and radar absorption and camouflage.

Metals or metal-coated materials generally show very high EMI-SE, as discussed in the previous section; however, they cannot be used as electromagnetic wave absorbers since high conductivity makes them shield by surface reflection. On the other hand, intrinsically conducting polymers are able to absorb as well as reflect electromagnetic waves, exhibiting then a significant advantage over metallic materials.

Actually, the total EMI shielding effectiveness of a material is the sum of shielding effectiveness resulting from three shielding mechanisms: reflection, absorption and multiple reflections. The relative shielding efficiency by reflection and absorption is determinant of practical application. Since EMI shielding by absorption rather than reflection can be more important for many applications, electrically conducting polymers can be preferred, because they are capable of reflecting and also of absorbing the electromagnetic wave and therefore exhibit a significant advantage over the metallic shielding materials. EMI shielding by absorption may be enhanced by increasing the skin depth, which is controlled by changing the electrical conductivities or the dielectric constants of electrically conducting polymers.

For EMI shielding applications, the polypyrrole (PPy) is known to possess high conductivity, easy synthesis and good environmental stability. For these reasons, one of the first commercial textile products based on conducting polymers was a fabric incorporating polypyrrole. It was the Contex ${ }^{\circledR}$, manufactured by Milliken \& Co., from 1990. The fabric was made by fibres coated with polypyrrole, woven to create an antistatic product. In fact, Milliken and Co. had been interested in using this type of antistatic technology for its carpet products. The material's best chance for success was in military applications, where the textile was used for ultralight camouflage netting to help conceal military equipment and personnel from near-infrared and radar detection (Patent US 8013776 B2). Today, textiles with $\mathrm{PPy} /$ polyester fibres are produced by Eeonyx Corp. as EeonTex ${ }^{\mathrm{TM}}$ conductive textiles. As in the case of Contex, these textiles are produced with individual fibres within a fabric or yarn completely and uniformly coated with doped polypyrrole.

The shielding effectiveness of some samples from EeonTex with different surface conductivity was measured by a dual-TEM cell [35]. The shielding effectiveness ranged between $5 \mathrm{~dB}$ and $20 \mathrm{~dB}$ from $0.2 \mathrm{GHz}$ to $0.8 \mathrm{GHz}$. In [35], it was observed that an increase of SE is obtained for high surface conductivity. A better conductivity produces reflection of waves, and the behaviour of the shield is going to be like that of metals. However, it was also observed that the shielding effectiveness increased also with frequency, due to an increase of absorption. This is in agreement with the results given in [36,37]. As discussed in these references, an enhanced variation in absorption as a function of frequency is observed for high resistance fabrics. However, the carbon-coated fabrics show this behaviour even at low resistances, thus highlighting one of the advantages of PPy-coated fabrics: in them, an enhanced absorption as a function of frequency is generally observed.

Other investigations on polypyrrole/polyester woven fabric have been done by Kim et al. [38]. They used chemical polymerization, where pyrrole dissolved in an aqueous solution was first sprayed on the polyester fabric and oxidized by spraying an aqueous solution of an oxidant and a dopant. They also performed a further electrochemical polymerization 
in an aqueous electrolyte solution, applying a constant current density to the polyester fabric coated with chemically polymerized PPy. The researchers investigated the effects of the chemical and of the electrochemical polymerization conditions on the properties of the resulting composite. Moreover, they observed that the composites shield EMI by absorption as well as reflection and that EMI shielding through reflection increases with the electrical conductivity (in [38], observed SE was of about $36 \mathrm{~dB}$ over a wide frequency range up to 1.5 $\mathrm{GHz}$ ). The relationship between SE and electrical conductivity of the PPy composite obtained in [38] coincides with the relationships reported for other conducting polymer systems such as those containing polyaniline in polyaniline/polymer composites [3943]. EMI SE of $36 \mathrm{~dB}$ of the PPy composite was comparable to or even higher than EMI SE reported for polyaniline systems or metal-coated carbon fibre composites.

As shown in [38], shielding efficiency by reflection increases with the decrease of the specific volume resistivity $\rho$. Contrarily, the shielding efficiency by absorption increases with the increase of $\rho$. The increase of reflection must be due to shallower skin depth of the composite with higher electrical conductivity. Again, we can conclude another important advantage of composites prepared with PPy: relative shielding efficiency by reflection and absorption can be easily controlled by controlling the electrical conductivity of PPy.

In [43], Hong et al. started to study the PPy/polyester complexes obtained through the chemical coating of polypyrrole doped with naphthalene sulfonic acid on polyester woven fabric. They coated this fabric with silver. The silver was thermally vacuum evaporated on the surface: then they measured EMI SE in the frequency range from $50 \mathrm{MHz}$ to $1.5 \mathrm{GHz}$ with ASTM D4935-99 method. The EMI SE of PPy/polyester complexes increases as the area of $\mathrm{Ag}$ evaporation layer increases. When the $\mathrm{Ag}$ is evaporated on one side of fabric complexes, the EMI $\mathrm{SE}$ is $33 \mathrm{~dB}$ at $0.5 \mathrm{GHz}$. The EMI SE of fabric without $\mathrm{Ag}$ is $27 \mathrm{~dB}$.

In [44], the researchers proposed a coating of polyester and polyethylene fabrics with $\mathrm{PPy}$ and $\mathrm{Ag}$ and Palladium, reaching shielding effectiveness from 65 till $80 \mathrm{~dB}$ in the range from 10 to $1000 \mathrm{MHz}$.

In [45], the dielectric characteristics of conducting polymer-coated textiles in the frequency range 1-18 $\mathrm{GHz}$ were investigated. Polypyrrole coatings were applied by solution polymerization on fabric substrates using a range of concentrations of paratoluene-2-sulfonic acid (pTSA) as dopant and ferric chloride as oxidant. The conducting polymer coatings exhibited dispersive permittivity behaviour with a decrease in real and imaginary components of complex permittivity as frequency increased in the range tested. Both the permittivity and the loss factor were affected by the polymerization time of the conductive coating. It was found that the total shielding efficiency of these conductive fabrics is significant at short polymerization times and increases to values exceeding $80 \%$ with longer polymerization times. The reflection contribution to electromagnetic shielding also increases with polymerization time.

\section{Effects of synthesis parameters and substrate treatments}

Actually, several publications in the area of conductive polymer coated textiles exist [46-51], which had investigated the resulting polymer conductivity. In particular, [46] is pointing out the role of the concentrations of the chemicals used in the polymerisation and of temperature and time in the final result. In [46], for instance, it is shown that the rate of polymerisation is strongly affected by the temperature of the synthesis.

Besides the effect of the synthesis parameters on coating, another important feature for applications is the persistence of the adhesion of polypyrrole to the substrates. For the textile industry, we have the plasma surface treatments which are able to modify the surface properties of inert polymeric materials, such as polyester. On textile surfaces, three main effects can be obtained depending on the treatment conditions: the cleaning effect, the increase of microroughness and the production of radicals to obtain hydrophilic surfaces. The advantage of plasma treatments is that the modification turns out to be restricted in the uppermost layers of the substrate, thus not affecting the overall desirable bulk properties [52-54].

In [55], the researchers have studied the effect of the atmospheric plasma glow discharge (APGD) treatment in the improvement of adhesion of conductive polypyrrole coating on wool and polyester fabrics. The fabrics were pre-treated with APGD, to improve the ability of the substrate to bond with anthraquinone-2-sulfonic acid doped conducting polypyrrole coating. The effect of the plasma treatment was analysed, by studying abrasion resistance, surface resistivity and reflectance.

In [56], the researchers systematically studied the improvement of binding of polypyrrole with PET thin films and fabrics using low pressure oxygen plasma. The study gives the result that both the increase in surface functionalisation by carboxylic groups and formation of nano-size roughness contributed to improved adhesion and conductivity. 
Also in [57], we can find researches on plasma treatments of polyester fabrics to increase polypyrrole/ $/ \mathrm{PW}_{12} \mathrm{O}_{40}{ }^{3-}$ adhesion. In the reference it is told that the roughness of the fibres increases as it has been observed by means of atomic force microscopy (AFM). Polar functional groups are also created on the surface of polyester fabrics as X-ray photoelectron spectroscopy (XPS) measurements have shown. These polar groups contribute to the adhesion of polypyrrole to the fibres. Coatings obtained on plasma treated fabrics were more resistant to washing and rubbing fastness tests. As explained in [57], the use of the counter ion $\left(\mathrm{PW}_{12} \mathrm{O}_{40}{ }^{3-}\right)$ that contains an element with a high atomic number (W) helps to locate the zones where the coating is missed. This control is achieved by means of micrographs obtained by backscattered electrons (BSE). The plasma treatment is also influencing the electrical resistance of the fabrics.

\section{Thermal electric effects and heat generation}

As we have previously seen, the PPy/polyester textiles are suitable for applications from antistatic films to electromagnetic interference shielding devices. However other applications exist, linked to the thermal-electric performance and the heat generation of these fabrics. As shown in [58], the measured thermal-electric Seebeck effect is comparable with that of metallic thermocouples. Since polypyrrole shows an extremely low thermal diffusivity regardless of its electrical conductivity, the low thermal conductivity gives significant advantage to the thermoelectric figure-of-merit ZT, comparable with that of some traditional inorganic thermoelectric materials. Let us remember that the figure-of-merit is a dimensionless quantity giving the ability of a material to efficiently produce thermoelectric power. In [58], the heat generation is also investigated for possible heating textile devices.

Also in [59], the researchers worked for the preparation and characterization of electrically conductive polyester textiles for heat generation. They used needle-punched nonwoven, spunlace nonwoven, and woven fabrics, which had been made electrically conductive by in situ chemical polymerization of pyrrole with p-toluene sulfonic acid dopant. The electro-conductive fabrics that they obtained are displaying an exponential rise of surface temperature on application of voltage. The rise of temperature was found to be related to the time duration of the applied voltage. The electroconductive fabrics exhibited linear voltage-current relationship at low voltage range. In [60], the heat generation of polypyrrole-coated polyacrylonitrile fibrous mats has been studied too.

\section{Biosignal monitoring}

As a consequence of its properties, $\mathrm{PPy} /$ polyester can be used for electronic textiles, that is, for preparing smart garments or smart textiles, which can contain digital components and allow electronics to be embedded in them. Here some references on the applications in smart textiles.

As observed in [61], an article which gives an overview of the technologies concerning textile integrated sensors and electrodes for monitoring of biosignals (electrocardiographic-ECG and electromyographic-EMG, breathing and moisture), it is possible to integrate sensors and electrodes directly into the fabrics, which can be used in clothing for monitoring people in several conditions. Let us consider electrodes for instance. Different design methods for preparing medical electrodes exist, among them we find also those based on conducting polymers. Then, aiming to obtain a proper electrode, in [62] a new synthetic route was reported for the synthesis and bonding of polypyrrole to a polyethylene terephthalate fabric. This route "involves a three-step process including surface phosphonylation and graft polymerization from the gaseous phase" [62]. The values of the dynamic surface wetting indicated that the polypyrrole-coated fabric is more hydrophilic than the untreated control. Because of a surface resistivity in the range $10^{4}-10^{5}$ $\Omega /$ square, the authors of [62] consider the polypyrrole-coated fabrics as attractive candidates for biomedical applications.

In [63], for the monitoring of biosignals the researches have proposed the use of a textile material which is acting as a sensor too. Reference 63 describes the applications of conductive polymer coated textiles, particularly polypyrrole, in wearable medical monitoring systems.

Also in [64], the design methods and classification of medical electrodes are discussed. In the article, the many methods that can be used to prepare fabric electrodes are reviewed, in order to get light fabric electrodes which have high conductivity and good conformability. As stressed in [64], "as a new generation of bio-signal acquisition material, fabric electrode has met the requirements of the development of modern medicine. Fabric electrode is different from traditional conductive materials in the characteristics of comfort, intelligence, convenience, accuracy and so on".

In [65], we find a review which summarizes the basic principles and approaches employed for having the fabric sensors. The most commonly used materials and techniques are also discussed. "The current work demonstrates that fabric sensors can be tailored to measure force, pressure, chemicals, humidity and 
temperature variations". In [65], the polypyrrole, in polypyrrole-coated Lycra fibres, is mentioned for having strain fabric sensors. It is also used for the detection of chemicals and gases in chemo-resistors, that is, in sensors whose electric resistance is sensitive to the chemical environment. " $\mathrm{H}_{2}$ and $\mathrm{CO}$ can be detected using conducting polymers doped with metallic inclusions; polypyrrole doped with copper and palladium inclusions shows a change in resistance when exposed to these gases" $[65,66]$.

An example of the use of polypyrrole as a strain fabric bio-sensor is given also in [67]. In this reference, the sensor is made of polypyrrole chemically deposited on a highly elastic fabric. This fabric produces a reproducible change in electrical resistance on stretching. "The resistance response to stretching is linear in $20 \%-40 \%$ strain variation" [67].

\section{Biocompatibility and biomedical applications}

Due to a possible large use of polypyrrole coated polyester, it is natural to discuss the investigations made on the biological compatibility of polypyrrole. In Reference 68 for instance, electrically conducting polypyrrole-treated films have been shown to influence the morphology and function of mammalian cells in vitro. Such a research is important because this type of polymer represents a possible alternative biomaterial for use in vascular implantation. The study in [68] compared the in vitro biocompatibility of five different polyester woven fabrics having increasing levels of electrical conductivity. The results of the study indicated that high levels of conductivity, that is, with surface resistance less than $200 \Omega$ /square on polyester fabrics, are "detrimental to the growth, migration, and viability of endothelial cells" [68].

In [69], we find a study that investigated the basic biocompatibility aspects of two types of polypyrrole (PPy)-coated polyester fabrics for possible use as vascular prostheses. These PPy-coated fabrics are PPy-Phos (PPy prepared using phosphonylation) and PPy-Plas (prepared using plasma activation). As told in [69], the "PPy-coated fabrics revealed different surface morphologies by showing more PPy lamina and clusters on the PPy-Plas. Neither of the PPycoated fabrics had an adverse effect on hemolysis and coagulation time, and they did not cause any acute systemic toxicity ... These results therefore highlighted the potential of PPy-coated fabrics for use as cardiovascular prostheses. It was suggested that cell adhesion moieties should be incorporated into the PPy/fabric composite to increase cell adhesion and subsequent cell proliferation".

In [70], the in vivo behaviours of polypyrrole for tissue engineering had been investigated, in particular the in vivo biocompatibility and biostability of PPy- coated polyester fabrics. The specimens were implanted subcutaneously in the back of rats for 3-90 days, then harvested and processed for enzymatic, histological, and morphological analyses. "This experiment suggests a similar in vivo biocompatibility of the PPy-coated and noncoated polyester fabrics and the importance of achieving a thin, uniform PPy coating" [70].

In [71], it is remarked that the "current conductive materials for use in cardiac regeneration are limited by cytotoxicity or cost in implementation". The researches in [71] demonstrate the application of a biocompatible, conductive polypyrrolepolycaprolactone (PPy-PCL) film "as a platform for culturing cardiomyocytes for cardiac regeneration". The aim of the researchers was that of finding a polymer having the potential to enhance engineered cardiac tissue function. The PPy-PCL seems suitable as an electrically-conductive substrate for culture of cardiomyocytes. We can conclude as in [71], that the results obtained using this PPy-based material encourage further development of conductive substrates for use in bio-engineering applications.

\section{Appendix A: Mechanisms behind the electromagnetic shielding}

The mechanisms behind the electromagnetic shielding had been discussed in details in [35], then we repeat here just a few points. Shielding electromagnetic radiation theory is based on two fundamental mechanisms, the reflection loss and the absorption loss. Let us imagine to place a metallic barrier in the path of an electromagnetic plane wave. The electric and magnetic field components, $\mathrm{E}$ and $\mathrm{H}$ respectively, are related by the wave impedance $\mathrm{Z}=$ $\mathrm{E} / \mathrm{H}$. In the free space, wave impedance is $\mathrm{Z}_{\mathrm{o}}=377$

$\Omega$. However, a metallic screen has a much lower impedance, given by $Z_{S}=(\omega \mu / \sigma)^{1 / 2}$, where $\mu$ is the permeability, $\sigma$ the conductivity and $\omega$ the angular frequency. This implies an impedance mismatch for the wave, which causes its reflection. The remaining field is transmitted through the barrier after partial absorption by the metal. The electric field component is reflected predominantly when it has to pass into a medium with lower impedance (front surface), while the magnetic component is reflected when it must pass into a medium with higher impedance (rear surface). Between the two surfaces there will be multiple reflections, which may be overlooked if the absorption loss in the barrier is at least $10 \mathrm{~dB}$.

The absorption loss, $\mathrm{S}_{\mathrm{AdB}}$, commonly expressed in $\mathrm{dB}$, is proportional to the shield thickness and increases with frequency. The reflection loss, $\mathrm{S}_{\mathrm{RdB}}$, meanwhile, decreases with frequency. The total loss, neglecting multiple reflections in the shield, is $\mathrm{S}=$ $\mathrm{S}_{\mathrm{AdB}}+\mathrm{S}_{\mathrm{RdB}}$. The sum of all the losses, reflection, 
absorption, but also multiple reflections, gives the shielding effectiveness SE. In the case of a multiplelaminated shield, the total reflection and absorption losses may be seen as the sum of reflection losses at each surface and the sum of attenuation losses in each layer.

We can consider the materials for shielding (not only metals), according to the shielding mechanisms. If the mechanism of EMI shielding we are considering is reflection, to reflect radiations the shield must have mobile charge carriers (electrons or holes) which interact with the electromagnetic fields in the radiation. As a result, the shield tends to be electrically conducting, although a high conductivity is not required since a volume resistivity of the order of $1 \Omega . c m$ is typically sufficient. Electrical conductivity is not a criterion for shielding, as conduction requires connectivity in conduction paths or percolation in the case of materials containing a conductive filler, whereas shielding does not.

Although shielding does not require connectivity, it is enhanced by connectivity. Metals are by far the most common materials for EMI shielding, working mainly by reflection due to the free electrons in them. Metal coatings made by electroplating, electroless plating, or vacuum deposition are commonly used for shielding [72,73]. The coatings may be on bulk materials, fibres or particles. However, coatings tend to suffer from their poor wear or scratch resistance. The secondary mechanism of EMI shielding is absorption. For a significant absorption by the shield, it should have electric and/or magnetic dipoles interacting with the electromagnetic radiation [74]. Then, the absorption loss is a function of the product $\sigma \mu$, whereas the reflection loss is a function of the ratio $\sigma / \mu$. In this manner, silver, copper, gold and aluminium are excellent for reflection, due to their high conductivity. Super-permalloy and mu-metal, which are nickel-iron soft magnetic alloys, are excellent for absorption, due to their high magnetic permeability.

Another mechanism of shielding is given by the multiple reflections, referring to the reflections at various surfaces or interfaces in the shield. This mechanism requires the presence of a large surface area or interface area in the shield that can be achieved, for example, in porous or foam material (large surface) or in composite material containing fillers which have a large surface area (large interface).

The loss due to multiple reflections can be neglected when the distance between the reflecting surfaces or interfaces is large as compared to the skin depth. Let us remember that the electromagnetic radiation at high frequencies penetrates only the near surface region of an electrical conductor, according to the skin effect. The penetration depth, known as skin depth $\delta$, decreases with increasing frequency and with increasing conductivity or permeability. In aluminium, $\delta=12 \mathrm{~mm}$ at $50 \mathrm{~Hz}$ and $\delta=0.1 \mathrm{~mm}$ at 1 MHz.

\section{Appendix B: Attenuation measurements}

Attenuation of the electromagnetic energy caused by a material may be characterised, depending on the measuring method used, by two quantities: the screening effectiveness, SE, and the insertion loss, A. The screening effectiveness is defined as the ratio of electromagnetic field strength $\mathrm{E}_{0}$ measured without and with the tested material $\mathrm{E}_{1}$ when it separates the field source and the receptor: $\mathrm{SE}_{\mathrm{dB}}=20 \log \mathrm{E}_{0} / \mathrm{E}_{1}$. It is dependent on the distance between the source and receptor of electromagnetic energy but in the far field zone, it characterises the attenuation of the electromagnetic wave in TEM-cells [35]. The measurement carried out in the near field zone characterises the effectiveness for the electric or magnetic field component only, depending on the antennae used as source and receptor.

The insertion loss $\mathrm{A}$ is a measure of the losses (or attenuation) of a transmitted signal caused by the tested material being inserted into the measuring channel: $A_{d B}=10 \log U_{0} / U_{1}$, being $U_{0}$ the channel output voltage without the tested material and $U_{1}$ the same voltage with the tested material [75].

Let us conclude noting that the methods used for estimating the shielding effectiveness of screened rooms are well-known and have been subjected to standardisation [76,77], but the measurement of the screening effectiveness or insertion loss of plastic or fabrics is rather a difficult topic, and has not up to now been standardised. Many measuring methods have been developed and are used in various laboratories. They differ in frequency range, sample dimensions, measurement conditions etc. We used for instance a dual TEM-cell [78-80], in which the screening effectiveness (SE) is measured.

\section{References}

1. Kuhn, H. H. (1993). Characterization and application of polypyrrole-coated textiles. In Intrinsically Conducting Polymers: An Emerging Technology (pp. 25-34). Springer Netherlands. DOI: DOI: 10.1007/978-94-017-1952-0_3

2. Smith, W. C. (2010). Smart textile coatings and laminates. Elsevier. ISBN: 1845697782, 9781845697785

3. Buckley, R. W. (2003). Polymer enhancement of technical textiles. iSmithers Rapra Publishing. ISBN: 1859573959, 9781859573952

4. Roshan, P. (2014). Functional finishes for textiles: Improving comfort, performance and protection. Elsevier. ISBN: 0857098454, 9780857098450

5. Skotheim, T. A. (1997). Handbook of Conducting Polymers, Second Edition, CRC Press. ISBN: 0824700503, 9780824700508 
6. Ji, Li Na (2013). Study on preparation process and properties of polyethylene terephthalate (PET). Applied Mechanics and Materials. $\quad 312, \quad 406-410 . \quad$ DOI: 10.4028/www.scientific.net/AMM.312.406

7. Schuler, M. J. (1981). Dyeing primer. Part 8: Dyeing with disperse dyes. AATCC. Research Triangle Park, NC, USA, p. 21.

8. Vernitskaya, T. V., \& Efimov, O. N. (1997). Polypyrrole: a conducting polymer, its synthesis, properties and applications. Russian Chemical Reviews, 66 (5), 443-457. DOI: 10.1070/RC1997v066n05ABEH000261

9. McNeill, R., Siudak, R., Wardlaw, J. H., \& Weiss, D. E. (1963). Electronic conduction in polymers. I. The Chemical Structure of Polypyrrole. Aust. J. Chem. 16, 1056-75. DOI: $10.1071 /$ CH9631056.

10. Heeger, A. (2000). Semiconducting and metallic polymers: The fourth generation of polymeric materials. Nobel Lecture, December 8, 2000, Aula Magna, Stockholm University. DOI: 10.1002/1521-3773(20010716)40:14<2591::AIDANIE2591>3.0.CO;2-0

11. MacDiarmid, A. G. (2000). Synthetic metals: A novel role for organic polymers. Nobel Lecture, December 8, 2000, Aula Magna, Stockholm University. DOI: DOI: 10.1002/1521-3773(20010716)40:14<2581::AIDANIE2581>3.0.CO;2-2

12. Shirakawa, H. (2000). The discovery of polyacetylene film: The dawning of an era of conducting polymers. Nobel Lecture, December 8, 2000, Aula Magna, Stockholm University. $\quad$ DOI: $10.1002 / 1521$ 3773(20010716)40:14<2574::AID-ANIE2574>3.0.CO;2-N

13. Kasevich, R. S. (2002). Cellphones, radars, and health, Spectrum IEEE, 39, 15-16. DOI: 10.1109/MSPEC.2002.1021945

14. Hemming, L. H. (2000). Architectural electromagnetic shielding handbook: A design and specification guide. Wiley-IEEE press. ISBN: 0780360249,9780780360242

15. Sparavigna, A. C. (2016). Engineered polymers for preventing electrostatic discharge in packaging. SSRN Electronic Journal, Paper n.2833724. DOI: $10.2139 /$ ssrn.2833724

16. Dordevic, Z. (1992). Textile fabric shielding electromagnetic radiation and clothing made thereof. Finex-Handels-Gmbh. Patent US5103504 A

17. Last, B. J., \& Thouless, D. J. (1971), Percolation theory and electrical conductivity. Phys. Rev. Lett. 27, 1719-1721. DOI: 10.1103/PhysRevLett.27.1719

18. Miyasaka, K. (1986). Mechanism of electrical conduction in electrically-conductive filler-polymer composites. Int. Polym. Sci. Technol., 13, 41-48.

19. Simon, R. M., \& Stutz, D. (1983). Test methods for shielding materials. EMC Technol. 2 (4), 39-48.

20. Martin, P. M. (2009). Handbook of deposition technologies for films and coatings: science, applications and technology. William Andrew. ISBN: 0815520328, 9780815520320

21. Bishop, C. (2011). Vacuum deposition onto webs, films and foils. William Andrew. ISBN: 1437778682, 9781437778687

22. Huang, J. C. (1995). EMI shielding plastics: A review. Advances in polymer technology, 14 (2), 137-150. DOI: 10.1002/adv.1995.060140205

23. Han, E. G., Kim, E. A., \& Oh, K. W. (2001). Electromagnetic interference shielding effectiveness of electroless $\mathrm{Cu}$-plated PET fabrics. Synthetic Metals, 123 (3), 469-476. DOI: 10.1016/S0379-6779(01)00332-0

24. Lu, Y., Jiang, S., \& Huang, Y. (2010). Ultrasonic-assisted electroless deposition of $\mathrm{Ag}$ on PET fabric with low silver content for EMI shielding. Surface and Coatings Technology, 204 (16), 2829-2833. DOI: 10.1016/j.surfcoat.2010.02.061

25. Lili, L., Dan, Y., Le, W., \& Wei, W. (2012). Electroless silver plating on the PET fabrics modified with 3-mercaptopropyltriethoxysilane. Journal of Applied Polymer Science, 124 (3), 1912-1918. DOI: 10.1002/app.35100

26. Xueping, G., Yating, W., Lei, L., Bin, S., \& Wenbin, H. (2008). Electroless plating of Cu-Ni-P alloy on PET fabrics and effect of plating parameters on the properties of conductive fabrics. Journal of Alloys and Compounds, 455 (1-2), 308-313. DOI: 10.1016/j.jallcom.2007.01.054

27. Gan, X. P., Wu, Y. T., Hu, W. B., \& Tang, Y. W. (2007) Study on properties of conductive PET fabrics prepared by electroless Copper and Nickel plating. Journal of Materials $\begin{array}{llll}\text { Engineering, } & 8, & 002 . & \text { URL: }\end{array}$ http://en.cnki.com.cn/Article_en/CJFDTOTALCLGC200708002.htm

28. See report given http://media.digikey.com/pdf/data\%20sheets/laird\%20techno logies/emi-sample-kit.pdf.

29. Henn, A. R., \& Cribb, R. M. (1992). Modeling the shielding effectiveness of metallized fabrics. International Symposium on Electromagnetic Compatibility IEEE. DOI: 10.1109/isemc.1992.626095

30. Henn, A. R. (1996). Interference Technology Engineering Master (ITEM) Update, p. 66-72.

31. Lai, K., Sun, R. J., Chen, M. Y., Wu, H., \& Zha, A. X. (2007). Electromagnetic shielding effectiveness of fabrics with metallized polyester filaments. Textile Research Journal, 77 (4), 242-246. DOI: 10.1177/0040517507074033

32. Ortlek, H. G., Saracoglu, O. G., Saritas, O., \& Bilgin, S. (2012). Electromagnetic shielding characteristics of woven fabrics made of hybrid yarns containing metal wire. Fibers and polymers, 13 (1), 63-67. DOI: 10.1007/s12221-0120063-6

33. Su, C. I., \& Chern, J. T. (2004). Effect of stainless steelcontaining fabrics on electromagnetic shielding effectiveness. Textile Research Journal, 74 (1), 51-54. DOI: 10.1177/004051750407400109

34. Goosey, M. (2012). Plastics for electronics. Springer Science \& Business Media. ISBN: 9400949421, 9789400949423.

35. Sparavigna A., Henn, A.R., \& Florio, L. (2005). Textiles as electromagnetic shields for human and device safety. In Applied Physics, Recent Res. Develop., pp. 1-20. ISBN: 9788178951874

36. Wong, P. T. C., Chambers, B., Anderson, A. P., \& Wright, P. V. (1992). Large area conducting polymer composites and their use in microwave absorbing material. Electronics letters, 28 (17), 1651-1653. DOI: 10.1049/el:19921051

37. Wright, P. V., Wong, T. C. P., Chambers, B., \& Anderson, A. P. (1994). Electrical characteristics of polypyrrole composites at microwave frequencies. Advanced Functional Materials, 4 (4), 253-263. DOI: 10.1002/amo.860040404

38. Kim, M. S., Kim, H. K., Byun, S. W., Jeong, S. H., Hong, Y. K., Joo, J. S., Song, K. T., Kim, J. K., Lee, C. J., \& Lee, J. Y. (2002). PET fabric/polypyrrole composite with high electrical conductivity for EMI shielding. Synthetic metals, 126 (2), 233-239. DOI: 10.1016/S0379-6779(01)00562-8

39. Chandrasekhar, P., \& Naishadham, K. (1999). Broadband microwave absorption and shielding properties of a poly (aniline). Synthetic metals, 105 (2), 115-120. DOI: 10.1016/S0379-6779(99)00085-5

40. Wessling, B. (1998). Dispersion as the link between basic research and commercial applications of conductive polymers (polyaniline). Synthetic Metals, 93 (2), 143-154. DOI: 10.1016/S0379-6779(98)00017-4

41. Koul, S., Chandra, R., \& Dhawan, S. K. (2000). Conducting polyaniline composite for ESD and EMI at $101 \mathrm{GHz}$ Polymer, 41 (26), 9305-9310. DOI: 10.1016/S00323861(00)00340-2

42. Gangopadhyay, R., De, A., \& Ghosh, G. (2001). Polyanilinepoly (vinyl alcohol) conducting composite: material with easy processability and novel application potential. Synthetic Metals, 123 (1), 21-31. DOI: 10.1016/S0379-6779(00)005737

43. Hong, Y. K., Lee, C. Y., Jeong, C. K., Sim, J. H., Kim, K., Joo, J., Kim, M. S., Lee, J. Y., Jeong, S. H., \& Byun, S. W. (2001). Electromagnetic interference shielding characteristics of fabric complexes coated with conductive polypyrrole and thermally evaporated Ag. Current App. Physics, 1 (6), 439-442.

44. Lee, C. Y., Lee, D. E., Jeong, C. K., Hong, Y. K., Shim, J. H., Joo J., Kim, M. S., Lee, J. Y., Jeong, S. H., Byun, S. W., 
Zang, D. S. \& Yang, H. G. (2002). Electromagnetic interference shielding by using conductive polypyrrole and metal compound coated on fabrics. Polym. Adv. Technol. 13, 577-583. DOI: $10.1002 /$ pat.227

45. Håkansson, E., Amiet, A., \& Kaynak, A. (2006). Electromagnetic shielding properties of polypyrrole/polyester composites in the $1-18 \mathrm{GHz}$ frequency range. Synthetic metals, $\quad 156 \quad$ (14), $917-925 . \quad$ DOI: 10.1016/j.synthmet.2006.05.010

46. Kaynak, A. and Beltran, R. (2003), Effect of synthesis parameters on the electrical conductivity of polypyrrolecoated poly(ethylene terephthalate) fabrics. Polym. Int., 52, 1021-1026. DOI: $10.1002 /$ pi.1195

47. Kuhn, H. H., Child, A. D., \& Kimbrell, W. C. (1995). Toward real applications of conductive polymers. Synthetic Metals, 71 (1-3), 2139-2142. DOI: 10.1016/03796779(94)03198-F

48. Child, A. D., \& Kuhn, H. H. (1997). Enhancement of the thermal stability of chemically synthesized polypyrrole. Synthetic metals, 84 (1), 141-142. DOI: 10.1016/S03796779(97)80684-4

49. Appel, G., Y fantis, A., Göpel, W., \& Schmeißer, D. (1996). Highly conductive polypyrrole films on non-conductive substrates. Synth. Methods 83 (3), 197-200. DOI: 10.1016/s0379-6779(97)80079-3

50. Malinauskas, A. (2001). Chemical deposition of conducting polymers. Polymer 42 (9), 3957-3972: DOI: 10.1016/s00323861(00)00800-4

51. Macasaquit, A. C., \& Binag, C. A. (2010). Preparation of conducting polyester textile by in situ polymerization of pyrrole. Philippine J. Sci., 139, 189-196. ISSN: 0031 - 7683

52. Sparavigna, A. C., \& Wolf, R. A. (2008). Atmospheric plasma treatments in converting and textile industries. Lulu Enterprises, Inc, pp. 1-112. ISBN: 9781409211075

53. Sparavigna, A. (2008). Plasma treatment advantages for textiles. arXiv preprint arXiv:0801.3727.

54. Wolf, R., \& Sparavigna, A. C. (2010). Role of Plasma Surface Treatments on Wetting and Adhesion. Engineering, 2 (6), 397-402. DOI: 10.4236/eng.2010.26052

55. Garg, S., Hurren, C., \& Kaynak, A. (2007). Improvement of adhesion of conductive polypyrrole coating on wool and polyester fabrics using atmospheric plasma treatment. Synthetic metals, 157 (1), 41-47. DOI: 10.1016/j.synthmet.2006.12.004

56. Mehmood, T., Kaynak, A., Dai, X. J., Kouzani, A., Magniez, K., de Celis, D. R., Christopher J. Hurren, \& du Plessis, J. (2014). Study of oxygen plasma pre-treatment of polyester fabric for improved polypyrrole adhesion. Materials Chemistry and Physics, 143 (2), 668-675. DOI: 10.1016/j.matchemphys.2013.09.052

57. Molina, J., Oliveira, F. R., Souto, A. P., Esteves, M. F., Bonastre, J., \& Cases, F. (2013). Enhanced adhesion of polypyrrole/PW12O403- hybrid coatings on polyester fabrics. Journal of Applied Polymer Science, 129 (1), 422 433. DOI: $10.1002 /$ app. 38652

58. Sparavigna, A. C., Florio, L., Avloni, J., \& Henn, A. (2010). Polypyrrole coated PET fabrics for thermal applications. Materials Sciences and Applications, 1 (04), 253. DOI: 10.4236/msa.2010.14037

59. Maity, S., Chatterjee, A., Singh, B., \& Pal Singh, A. (2014). Polypyrrole based electro-conductive textiles for heat generation. The Journal of The Textile Institute, 105 (8), 887893. DOI: $10.1080 / 00405000.2013 .861149$

60. Oroumei, A., Tavanai, H., \& Morshed, M. (2012). Electrical resistance and heat generation of polypyrrole-coated polyacrylonitrile nanofibrous and regular fibrous mats. Polymers for Advanced Technologies, 23 (9), 1302-1310. DOI: $10.1002 /$ pat.2049

61. Carvalho, H., Catarino, A. P., Rocha, A., \& Postolache, O. (2014). Health monitoring using textile sensors and electrodes: An overview and integration of technologies. IEEE International Symposium on Medical Measurements and Applications (MeMeA), 2014, pp. 1-6. DOI: 10.1109/memea.2014.6860033
62. Tessier, D., Dao, L. H., Zhang, Z., King, M. W., \& Guidoin, R. (2000). Polymerization and surface analysis of electrically-conductive polypyrrole on surface-activated polyester fabrics for biomedical applications. Journal of Biomaterials Science, Polymer Edition, 11 (1), 87-99. DOI: $10.1163 / 156856200743517$

63. Muthukumar, N. \& Thilagavathi, G. (2012). Conductive polymer coated textiles for biosignals monitoring. International Journal of Textile and Fashion Technology (IJTFT), 2 (2), 1-9. ISSN: 2250-2378

64. Liu, Z., \& Liu, X.X. (2015). Progress on fabric electrodes used in ECG signals monitoring. Journal of Textile Science and Technology, 1, 110-117. DOI: 10.4236/jtst.2015.13012

65. Castano, L. M., \& Flatau, A. B. (2014). Smart fabric sensors and e-textile technologies: a review. Smart Materials and Structures, 23(5), 053001. DOI: 10.1088/09641726/23/5/053001

66. Torsi, L., Pezzuto, M., Siciliano, P., Rella, R., Sabbatini, L., Valli, L., \& Zambonin, P. G. (1998) Conducting polymers doped with metallic inclusions: new materials for gas sensors. Sensors Actuators B, 48, 362-7. DOI: 10.1016/s0925-4005(98)00058-6

67. Rajagopalan, S., Sawan, M., Ghafar-Zadeh, E., Savadogo, O., \& Chodavarapu, V. P. (2008). A polypyrrole-based strain sensor dedicated to measure bladder volume in patients with urinary dysfunction. Sensors, 2008, 8(8), 5081-5095; doi:10.3390/s8085081

68. Jakubiec, B., Marois, Y., Zhang, Z., Roy, R., Sigot-Luizard, M.-F., Dugré, F. J., King, M. W., Dao, L. H., Laroche, G., \& Guidoin, R. (1998). In vitro cellular response to polypyrrole-coated woven polyester fabrics: Potential benefits of electrical conductivity. Journal of Biomedical Materials Research Part A, 41 (4), 519-526. DOI: 10.1002/(SICI)1097-4636(19980915)41:4<519::AIDJBM2>3.0.CO;2-F

69. Zhang, Z., Roy, R., Dugré, F. J., Tessier, D., \& Dao, L. H. (2001). In vitro biocompatibility study of electrically conductive polypyrrole-coated polyester fabrics. Journal of biomedical materials research, 57 (1), 63-71. DOI: 10.1002/1097-4636(200110)57:1<63::AIDJBM1142>3.0.CO;2-L

70. Jiang, X., Marois, Y., Traoré, A., Tessier, D., Dao, L. H., Guidoin, R., \& Zhang, Z. (2002). Tissue reaction to polypyrrole-coated polyester fabrics: an in vivo study in rats. Tissue engineering, 8 (4), 635-647. DOI: $10.1089 / 107632702760240553$.

71. Spearman, B. S., Hodge, A. J., Porter, J. L., Hardy, J. G., Davis, Z. D., Xu, T., Zhang, X., Schmidt, C. E., Hamilton, M. C., Lipke, E. A. (2015). Conductive interpenetrating networks of polypyrrole and polycaprolactone encourage electrophysiological development of cardiac cells. Acta Biomater. 2015 Dec, 28, 109-20. DOI: 10.1016/j.actbio.2015.09.025

72. Mandich, N.V. (1994). EMI shielding by electroless plating of ABS plastics. Plating and Surface Finishing, 81 (10), 6063. ISSN: 0360-3164

73. Sidhu, A., Reike, J., Michelsen, U., Messinger, R., Habiger, E., \& Wolf, J. (1997). Metallization of plastics for shielding, IEEE Int. Symp. Electromagnetic Compatibility, IEEE, Piscataway, New Jersey, USA: IEEE, pp. 102-5. DOI: 10.1109/elmagc.1997.617094

74. Chung, D. D. L. (2001). Electromagnetic interference shielding effectiveness of carbon materials. Carbon, 39 (2), 279-285. DOI: 10.1016/s0008-6223(00)00184-6

75. Pietranik, M., \& Stawski, W. (2004). New Type of Textiles with Shielding Properties. Fibres \& Textiles in Eastern Europe, 12 (3), 47

76. Wilson, P. F., \& Ma, M. T. (1988). Techniques for measuring the electromagnetic shielding effectiveness of materials. II Near-field source simulation. IEEE Transactions on Electromagnetic Compatibility, 30 (3), 251-259. DOI: $10.1109 / 15.3303$

77. Wilson, P. F. (1988). A comparison between near-field shielding-effectiveness measurements based on coaxial 
dipoles and electrically small apertures. IEEE transactions on electromagnetic compatibility, 30 (1), 23-28. DOI: $10.1109 / 15.19884$

78. Avloni, J., Ouyang, M., Florio, L., Henn, A. R., \& Sparavigna, A. (2007). Shielding effectiveness evaluation of metallized and polypyrrole-coated fabrics. Journal of Thermoplastic Composite Materials, 20 (3), 241-254. DOI: 10.1177/0892705707076718

79. Avloni, J., Lau, R., Ouyang, M., Florio, L., Henn, A. R., \& Sparavigna, A. (2008). Polypyrrole-coated nonwovens for electromagnetic shielding. Journal of Industrial Textiles, 38 (1), 55-68. DOI: 10.1177/1528083707087834

80. Avloni, J., Florio, L., Henn, A. R., Lau, R., Ouyang, M., \&

Sparavigna, A. (2006). Electromagnetic shielding with polypyrrole-coated fabrics. arXiv preprint condmat/0608664. 